there considered, and if

$$
\begin{aligned}
& y_{1}, y_{2}, \ldots, y_{n}, \\
& z_{1}, y_{2}, \ldots, z_{n},
\end{aligned}
$$

are the values of the parameters corresponding to an arbitrary operation $T$ of $G$ and to its inverse $T^{-1}$, it may be shown, alnost exactly as on pp. 556,557 , that

$$
z_{i-1}=\frac{1}{n D_{y}} \frac{\partial D_{y}}{\partial y_{i}}
$$

Now, if the $y$ 's have arbitrary values, so also have the $z$ 's; and therefore the $n$ differential coefficients $\frac{\partial D_{y}}{\partial y_{i}}$ are linearly independent. Heuce, by (iv) of $\S 5$, every irreducible factor of $D_{y}$ enters into $D_{y}$ to a power equal to its degree; and, if there are $s$ such irreducible factors, $G$ is the direct product of $s$ general linear groups in $m_{1}, m_{2}, \ldots, m_{s}$ variables, where

$$
m_{1}^{2}+m_{2}^{2}+\ldots+m_{s}^{2}=n \text {. }
$$

This being the case, the self-conjugate operations of $G$ constitnte a sub-group of order $s$. But it had previously been proved that this sub-group had $r$ for its order. Hence $s=r$, and the variables may be chosen so that every operation of both $G$ and $G^{\prime}$ interchange them in the same $r$ sets, the number in each set being a square. These are the main results on which the remainder of the discussion turns.

The Propagation of Light in a Uniaxal Crystal. By A. W. Conway. Received November 5th, 1902. Read November 13th, 1902.

\title{
Introduction.
}

The following paper is an adaptation of the analysis used by Prof. A. F. H. Love ("Integration of the Equations of Propagation of Electric Waves," Phil. Trans., Series A, 1901) to the case of a uniaxal crystalline medium, together with some deductions from the 
general equations and applications to physical optics. $\$ \S 1,2$, and 3 are occupied in integrating the equations of motion in terms of certain houndary conditions. $\S \S 4$ and 5 trent of the direction of the vibration and the flow of energy. $\$ \S 6,7$, and 8 are applications of the general integral obtained in $\S 3$ to Huygens' principle for a crystalline medium with a plane face, and to the passage of parallel and divergent beams of light through a thin crystalline plate. A 'note is added on Huygens' principle, which was suggested by some of the results in $\S 6$.

\section{Particular Solutions.}

If the medium be considered to be magnetically isotropic, but to possess an axis of electric symmetry, and if the magnetic permeability be taken to be unity, the differential equations of wave propagation on the basis of the electromagnetic theory of light are

$$
\left.\begin{array}{rl}
a^{-2} \dot{X} & =\frac{\partial \underline{\gamma}}{\partial y}-\frac{\partial \beta}{\partial z} \\
a^{-2} \dot{Y} & =\frac{\partial \alpha}{\partial z}-\frac{\partial \gamma}{\partial x} \\
c^{-2} \dot{Z} & =\frac{\partial \beta}{\partial x}-\frac{\partial a}{\partial y} \\
-\dot{a} & =\frac{\partial Z}{\partial y}-\frac{\partial Y}{\partial z} \\
-\dot{\beta} & =\frac{\partial X}{\partial z}-\frac{\partial Z}{\partial x} \\
-\dot{\gamma} & =\frac{\partial Y}{\partial x}-\frac{\partial X}{\partial y}
\end{array}\right\}
$$

where $(X, Y, Z)$ and $(a, \beta, \gamma)$ denote the electric and magnetic forces respectively in electromagnetic units.

From these equations we can deduce

$$
\begin{gathered}
a^{-2} \frac{\partial X}{\partial x}+a^{-8} \frac{\partial Y}{\partial y}+c^{-2} \frac{\partial Z}{\partial z}=0 \\
\frac{\partial \alpha}{\partial x}+\frac{\partial \beta}{\partial y}+\frac{\partial \gamma}{\partial z}=0
\end{gathered}
$$

wherever these functions have no singularities. For light waves it 
is known that the constants $a$ and $c$ vary with the wave-lengtl. In what follows they will be taken to be constants, and the results obtained will then be applicable to the case of monochromatic light.

If we put $a=c$ in equations (1), we get the case of an isotropic medium. In this case there are two classes of solutions which are infinite only at the origin. The simplest of these solutions, from which all the others can be derived, are (a) the case of a Hertzian oscillator or vibrating electric doublet, and $(b)$ the case of a vibrating magnetic doublet.

If $F(t)$ and $f(t)$ are arbitrury functions which are uniform, finite, and continuous for all real values of $t$, and if we denote by $x$ the function

$$
\begin{aligned}
\int_{0}^{\sqrt{x^{3}+\nu^{2}}} \frac{a d \lambda}{\lambda}\left\{F\left(a^{-1} \sqrt{\lambda^{2}+\pi^{2}}+t\right)-F\left(\sqrt{c^{-2} \lambda^{2}+a^{-2} z^{2}}+t\right)\right. \\
\left.+f\left(a^{-1} \sqrt{\lambda^{2}+z^{2}}-t\right)-f\left(\sqrt{c^{-2} \lambda^{2}+a^{-2} z^{2}}-t\right)\right\}
\end{aligned}
$$

we can show that $\chi$ has no infinity for real values of $\sqrt{x^{2}+y^{2}}$ and $z$, and that near the $z$-axis it can be expanded in positive integral powers of $x^{2}+y^{2}$, and hence that expressions of the form $\frac{\partial^{l+m+n}}{\partial x^{l} \partial y^{m} \partial z^{n}} \chi$ have no singularity on the $z$-axis. We have also the relations

$$
\begin{aligned}
\frac{\partial^{2} x}{\partial x^{2}}+\frac{\partial^{2} x}{\partial y^{2}} & =\frac{\partial}{\partial t}\left\{\frac{F\left(a^{-1} \sqrt{x^{2}+y^{2}+z^{2}}+t\right)}{\sqrt{ } x^{2}+y^{2}+z^{2}}-\frac{a}{c^{2}} \frac{F\left[\sqrt{c^{-2}\left(x^{2}+y^{2}\right)+a^{-2} z^{2}}+t\right]}{\sqrt{c^{-2}\left(x^{2}+y^{2}\right)+a^{-2} z^{2}}}\right] \\
& \left.-\frac{f\left(a^{-1} \sqrt{x^{2}+y^{2}+z^{2}}-t\right)}{\sqrt{ } x^{2}+y^{2}+z^{2}}+\frac{a}{c^{2}} \frac{F\left[\sqrt{c^{-2}\left(x^{2}+y^{2}\right)+a^{-2} z^{2}}-t\right]}{\sqrt{c^{-2}\left(x^{2}+y^{2}\right)+a^{-2} z^{2}}}\right\}(2) \\
& =\phi-\psi,
\end{aligned}
$$

where $\phi$ denotes the part involving $\sqrt{x^{2}+y^{2}+z^{2}}$ and $\psi$ denotes the part involving $\sqrt{c^{-2}\left(x^{2}+y^{2}\right)+a^{-2} z^{2}}$,

$$
\begin{gathered}
\frac{\partial^{2} \phi}{\partial x^{2}}+\frac{\partial^{2} \phi}{\partial y^{2}}+\frac{\partial^{2} \phi}{\partial z^{3}}=a^{-2} \frac{\partial^{2} \phi}{\partial t^{2}} \\
a^{-2} \frac{\partial^{2} \psi}{\partial x^{2}}+a^{-2} \frac{\partial^{2} \psi}{\partial y^{2}}+c^{-2} \frac{\partial^{2} \psi}{\partial z^{2}}=a^{-2} c^{-2} \frac{\partial^{2} \psi}{\partial t^{2}} \\
\partial^{2} \chi \\
\frac{\partial z^{2}}{2}=a^{-2} \frac{\partial^{2} \chi}{\partial t^{2}}-\phi+\frac{c^{2}}{a^{2}} \psi
\end{gathered}
$$


1902.]

Using the above notation, let us consider the following values for the components of magnetic force :-

$$
\left.\begin{array}{l}
a_{1}=a^{-2} \frac{\partial^{3} \dot{\chi}}{\partial x \partial y \partial z} \\
\beta_{1}=a^{-2} \frac{\partial \dot{\phi}}{\partial z}-a^{-2} \frac{\partial^{3} \dot{x}}{\partial x^{3} \partial z} \\
\gamma_{1}=-a^{-2} \frac{\partial \dot{\phi}}{\partial y}
\end{array}\right\} .
$$

Using the equations (1), we find for the electric force

$$
\left.\begin{array}{l}
X_{1}=-\left(\frac{\partial^{2} \phi}{\partial y^{2}}+\frac{\partial^{2} \phi}{\partial z^{2}}\right)+\frac{\partial^{4} x}{\partial x^{2} \partial z^{2}} \\
Y_{1}=\frac{\partial^{3} \phi}{\partial x \partial y}+\frac{\partial^{4} x}{\partial x \partial y \partial z^{2}} \\
Z_{1}=\frac{c^{2}}{a^{4}} \frac{\partial^{2} \phi}{\partial x \partial z}-\frac{c^{2}}{a^{2}}\left(\frac{\partial^{4} x}{\partial x^{3} \partial z}+\frac{\partial^{4} x}{\partial \partial^{2} \partial y^{2} \partial z}\right)
\end{array}\right\},
$$

which take the simpler forms, on making use of equations (2), (3), and (5),

$$
\left.\begin{array}{l}
X_{1}=-a^{-2} \ddot{\phi}+\frac{c^{2}}{a^{2}} \frac{\partial^{2} \psi}{\partial x^{2}}+a^{-2} \frac{\partial^{2} \ddot{x}}{\partial x^{2}} \\
Y_{1}=\frac{c^{3}}{u^{2}} \frac{\partial^{2} \psi}{\partial x \partial y}+a^{-2} \frac{\partial^{\ddot{2} x}}{\partial x \partial y} \\
Z_{1}=\frac{c^{2}}{a^{2}} \frac{\partial^{2} \psi}{\partial x \partial z}
\end{array}\right\} .
$$

On taking the curl of $\left(Y_{1}, Y_{1}, Z_{1}\right)$ in the above forms we find that it is identical with $-\left(\dot{a}_{1}, \dot{\beta}_{1}, \dot{\gamma}_{1}\right)$. Hence these expressions satisfy equations (1). On examination of the terms it will be seen that they are of two kinds. The first kind involves arbitrary functions of $a^{-1} \sqrt{x^{2}+y^{2}+z^{2}} \pm t$, which refer evidently to a radiation travelling with a velocity $a$. The second kind involves arbitrary functions of $\sqrt{c^{-2}\left(x^{2}+y^{3}\right)+a^{-2} z^{2}} \pm t$, which refer to a radiation travelling with a velocity $\left[c^{-2}\left(l^{2}+m^{2}\right)+a^{-2} n^{2}\right]^{-\frac{1}{d}}$ in the direction $l, m, n$. Hence the wave surface corresponding to this disturbance consists of two shents. 
one being a sphere and the other a spheroid. To see the physical meaning of the expressions (6) and (7), we may let $F$ and $f$ be simply periodic functions with a long period, and we see that the disturbance is due to an electric doublet whose axis is along the axis of $x$. On letting $a=c$ the functions $\phi$ and $\psi$ become identical and $\chi$ ranishes, and we obtain the well known solution of Hertz.

In a similar manner the electric and magnetic vectors for a doublet along the axis of $y$ are given by the equations

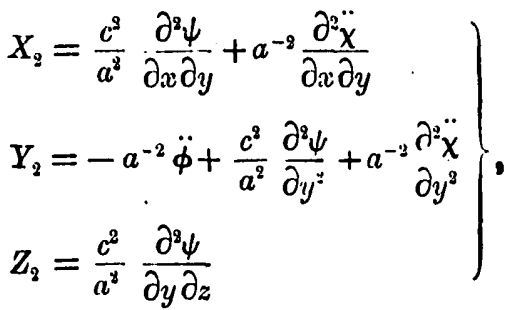

$$
\begin{aligned}
& \left.a_{3}=-a^{-2} \frac{\partial \dot{\phi}}{\partial z}+a^{-2} \frac{\partial^{3}}{\partial y^{3}} \frac{\dot{x}}{\partial z}\right) \\
& \left.\begin{array}{l}
\beta_{2}=-a^{-2} \frac{\partial^{3} \dot{x}}{\partial x \hat{\partial} y \partial z} \\
\gamma_{8}=a^{-2} \frac{\partial \dot{\dot{\phi}}}{\partial x}
\end{array}\right\}
\end{aligned}
$$

For an electric doublet along the axis of $z$ the components take the simpler forms

$$
\left.\begin{array}{l}
x_{3}=\frac{c^{3}}{a^{2}} \frac{\partial^{2} \psi}{\partial x \partial z} \\
Y_{3}=\frac{c^{2}}{a^{2}} \frac{\partial^{2} \psi}{\partial y \partial z} \\
Z_{3}=-\frac{c^{4}}{a^{4}}\left(\frac{\partial^{2} \psi}{\partial x^{2}}+\frac{\partial^{2} \psi}{\partial y^{2}}\right)
\end{array}\right\},
$$


It may be noticed that an electric doublet along the axis of $z$ gives rise only to a single wave surface-a spheroid-and it will be seen from the forms below (15) that a magnetic doublet along the same axis gives rise only to a spherical wave surface.

Besides electric doublets we can have magnetic doublets, which can also be regarded as currents flowing around small circuits. If the moment or the current varies with the time in any manner, a disturbance is propagated. We give below in (13), (14), and (15) the electric forces due to doublets of this kind having their axes along the axes of $x, y$, and $z$ respectively,

$$
\left.\begin{array}{l}
X_{1}^{\prime}=a^{-2} \frac{\partial^{3} x}{\partial x \partial y \partial z} \\
Y_{1}^{\prime}=-a^{-2} \frac{\partial \phi}{\partial z}+a^{-2} \frac{\partial^{y} x}{\partial y^{2} \partial \dot{z}} \\
Z_{1}^{\prime}=\frac{c^{2}}{a^{4}} \frac{\partial \psi}{\partial y}
\end{array}\right\} ;
$$

Introducing a vector-the magnetic displacement-which is connected with the magnetic force, thus

$$
\begin{aligned}
& \left(\dot{u}_{1} \dot{v}_{1} \dot{v}_{1}\right)=\left(a_{1} \beta_{1} \gamma_{1}\right), \\
& \left(\dot{u}_{3} \dot{v}_{3} \dot{u}_{3}\right)=\left(a_{2} \beta_{3} \gamma_{2}\right), \\
& \left(\dot{u}_{3} \dot{v}_{3} u_{3}\right)=\left(a_{3} \beta_{3} \gamma_{3}\right),
\end{aligned}
$$

we see that the expressions $X_{1}, Y_{1}, Z_{1}, u_{1}, v_{1}, v_{1}$ are identical with vol. $x \times x$. - No. 804 . 
$X_{1}, X_{3}, X_{8}, X_{1}^{\prime}, X_{2,}^{\prime}, X_{3}^{\prime}$, respectively ; and that $X_{9}, Y_{2}, Z_{2}, u_{9}, v_{1}, w_{1}$ are identical with $Y_{1}, Y_{2}, Y_{3}, Y_{1}^{\prime}, Y_{2}^{\prime}, Y_{3}^{\prime}$, respectively ; and that $X_{3}, Y_{8}, Z_{3}$, $u_{3}, v_{3}, w_{3}$ are identical with $Z_{1}, Z_{2}, Z_{3}, Z_{1}^{\prime}, Z_{2}^{\prime}, Z_{3}^{\prime}$, respectively.

\section{The Reciprocal Theorem.}

If $U$ and $\dot{V}$ satisfy the relations

$$
\nabla^{8} U=0, \quad \nabla^{2} V=0,
$$

it was shown by Green that

$$
\iint\left\{U \frac{\partial V}{\partial n}-V \frac{\partial U}{\partial n}\right\} d S=0,
$$

provided that the surface integrals are taken over the boundary of a region not containing singularities of $U$ and $V$. Similar theorems have been proved by Poisson and Kirchhoff for solutions of

$$
a^{2} \nabla^{2} U=\frac{\partial^{2} U}{\partial t^{2}}
$$

For the equations (1), if $(X, Y, Z),\left(X^{\prime}, Y^{\prime}, Z^{\prime}\right)$ be two possible solutions of the same period, we can verify the theorem

$$
\begin{aligned}
\iint\left\{X \frac{\partial X^{\prime}}{\partial n}-X^{\prime}\right. & \frac{\partial X}{\partial n}+Y \frac{\partial Y^{\prime}}{\partial n}-Y^{\prime} \frac{\partial Y}{\partial n}+Z \frac{\partial Z^{\prime}}{\partial n}-Z^{\prime} \frac{\partial Z}{\partial n} \\
& -(l X+m Y+n Z)\left(\frac{\partial X^{\prime}}{\partial x}+\frac{\partial Y^{\prime}}{\partial y}+\frac{\partial Z^{\prime}}{\partial z}\right) \\
& \left.+\left(l X^{\prime}+m Y^{\prime}+n Z^{\prime}\right)\left(\frac{\partial \dot{X}}{\partial x}+\frac{\partial Y}{\partial y}+\frac{\partial Z}{\partial z}\right)\right\} d S=0 .
\end{aligned}
$$

By taking $\quad V=\left\{\left(x-x^{\prime}\right)^{9}+\left(y-y^{\prime}\right)^{2}+\left(z-z^{\prime}\right)^{2}\right\}^{-1}$,

Green has shown that

$$
. \iint\left\{\cdot V \frac{\partial V}{\partial i}-V \frac{\partial U}{\partial i}\right\} d S=4 \pi D^{\prime},
$$

where $U^{\prime}$ is the value of $U$ at a point $x^{\prime}, y^{\prime}, z^{\prime}$ outside the boundary which encloses the singularities of $U$. This equation can be interpreted in the theory of electrostatics and of hydrodynamics, just as the theorems of Kirchhoff and Poisson have interpretations in the theory of sound. In like manner, by properly choosing $X^{\prime}, Y^{\prime}, Z^{\prime}$, we might, by the aid of (16), obtain the value of the electric force 
at an external point in terms of a surface integral. Such a theorem would be open to the objections urged by Prof. A. E. H. Love.* We shall therefore make use of the reciprocal theorem given by him.

Let $(X, Y, Z),(u, v, w)$ and $\left(X^{\prime}, Y^{\prime}, Z^{\prime}\right),\left(u^{\prime}, v^{\prime}, w^{\prime}\right)$ be two solutions of equation (1). Then, if the surface integration extends over the boundary of a region which is free from the singularities of these functions,

$$
\begin{aligned}
\int_{t_{0}}^{t_{1}} d t \iint\left\{l\left(v Z^{\prime}-v^{\prime} Z-w Y^{\prime}+w^{\prime} Y\right)\right. & +m\left(v X^{\prime}-w^{\prime} X-u Z^{\prime}+u^{\prime} Z\right) \\
& \left.+u\left(u Y^{\prime}-u^{\prime} Y-v X^{\prime}+v^{\prime} X\right)\right\} d S \\
= & \int_{t_{0}}^{t_{1}} d t \iiint d \tau\left\{X^{\prime}\left(\frac{\partial w}{\partial y}-\frac{\partial v}{\partial z}\right)-X\left(\frac{\partial w^{\prime}}{\partial y}-\frac{\partial v^{\prime}}{\partial z}\right)+u^{\prime}\left(\frac{\partial Z}{\partial y}-\frac{\partial Y}{\partial z}\right)-\ldots\right\} .
\end{aligned}
$$

But

$$
\begin{aligned}
& \frac{\partial v}{\partial y}-\frac{\partial v}{\partial z}=u^{-2} X \\
& \frac{\partial Z}{\partial y}-\frac{\partial Y}{\partial z}=-\dot{a}=-\ddot{u}
\end{aligned}
$$

therefore the expression beconres, on changing the order of integration,

$$
=\iiint d \tau\left[\dot{u} u i^{\prime}-u \dot{u}^{\prime}+\dot{v} v^{\prime}-\dot{v} \dot{v}^{\prime}+\dot{w} v v^{\prime}-v \dot{v} \dot{w}^{\prime}\right]_{t_{0}}^{t_{1}}=0
$$

if we take $u^{\prime}, v^{\prime}, w^{\prime}$ to be insensible at the times $t_{0}, t_{1}$.

\section{The Integration of the Equations and Huygens' Principle.}

Let us take for $\left(X^{\prime}, Y^{\prime}, Z^{\prime}\right),\left(u^{\prime}, v^{\prime}, v^{\prime}\right)$ the forms $\left(X_{1}, Y_{1}, Z_{1}\right)$, $\left(u_{1}, v_{1}, w_{1}\right)$ given in (6) and (7). We have at our disposal two arbitrary functions $F$ and $f$. Let $f=0$, and let

$$
\frac{\partial F(x)}{\partial x}=\zeta(x)
$$

Then we shall give $\zeta(x)$ the following two properties. $\zeta(x)$ is very nearly zero except in the interval from $-\eta_{0}$ to $\eta_{1}$, where $\eta_{0}$ and $\eta_{1}$ are

" "The Integration of the Equations of Propagation of Electric Waves," Phil. Trans., Series A, 1901, from which this section and the next are adapted. 
very small positive quantities, and

$$
\int_{-\eta_{0}}^{\eta_{1}} \zeta(x) d x=1
$$

If $\epsilon$ is a small positive number, and $x, y, z$ refer to any point on the small spheroid

$$
\frac{x^{2}}{c^{8}}+\frac{y^{2}}{c^{2}}+\frac{z^{3}}{a^{2}}=\epsilon^{2}
$$

we can find $t_{1}$ such that

$$
a^{-1} \sqrt{a^{2}+y^{2}+z^{2}}+t_{2} \text { and } \sqrt{c^{-2}}\left(x^{2}+y^{2}\right)+a^{-2} z^{2}+t_{1}>\eta_{1},
$$

and $t_{0}$ such that

$$
a^{-1} \sqrt{x^{2}+y^{2}}+z^{2}+t_{0} \text { and } \sqrt{ } c^{-2}\left(x^{2}+y^{2}\right)+a^{-2} z^{9}+t_{0}>-\eta_{0} .
$$

When $\quad \int^{t_{1}} \zeta\left(a^{-1} \sqrt{k^{3}+y^{2}+z^{2}}+t\right) d t=\int_{-\eta_{0}}^{\eta_{1}} \zeta(x) d x=1$,

$$
\begin{aligned}
& \int_{t_{0}}^{t_{1}} \sqrt{x^{2}+y^{2}+z^{2}} \zeta^{\prime}\left(t^{-1} \sqrt{x^{2}+y^{2}+z^{2}}+t\right) d t \\
& =\sqrt{x^{2}+y^{3}+z^{2}} \int_{t_{0}}^{t_{1}} \zeta\left(a^{-1} \sqrt{x^{2}+y^{2}+z^{8}}+t\right) d t=0,
\end{aligned}
$$

if $\sqrt{x^{3}+y^{9}+z^{9}}$ is sufficiently small, and similar relations hold for $\zeta\left\{\sqrt{c^{-1}\left(x^{2}+y^{2}\right)+a^{-3} z^{3}}+t\right\}$. Making use of this function $\zeta()$, it is clear that (17) is satisfied. An example of a function fulfilling these conditions is

$$
\zeta(x)=\frac{\mu}{\sqrt{ } \pi} e^{-\mu^{2} x^{2}}
$$

provided that $\mu$ is very large and $\epsilon$ is small of order $\mu^{-6}$. The surface of integration in (17) is a surface $\left(S_{1}\right)$ surrounding the singularities of $(X, Y, Z),(u, u, w)$, and a surface $\left(S_{9}\right)$ surrounding the origin which is the only singularity of $\left(X_{1}, X_{1}, Z_{1}\right),\left(u_{1}, v_{1}, v_{1}\right)$. This latter surface we shall take to be the small spheroid

$$
\frac{a^{2}}{c^{2}}+\frac{y^{3}}{c^{2}}+\frac{z^{3}}{a^{2}}=\epsilon^{2}
$$

We shall proceed now to find the value of the integral on the lefthand side of (17) when taken over $S_{y}$. Referring to (7) and (8), consider first the terms arising from differentiations of the function $\chi$. 
1902.] . Propagation of Light in a. Uniaxal Crystal.

They are

$$
\begin{aligned}
\int_{t_{0}}^{t_{1}} d t \iint d S\left\{a \cdot \frac{\partial^{2} \ddot{x}}{\partial x^{2}}(m u-n v)+a^{-2} \frac{\partial^{\ddot{3} x}}{\partial x \partial y}(n u-l w)\right. \\
\left.+a^{-2} \frac{\partial^{3} x}{\partial x \partial y \partial z}(m Z-n Y)-a^{\cdot 2} \frac{\partial^{3} X}{\partial x^{2} \partial z}(n X-l Z)\right\} .
\end{aligned}
$$

On integrating with respect to $t$ and letting $\epsilon$ become very small, we find that the only term which does not vanish is

$$
-\int_{t_{0}}^{t_{1}} d t \iint d S a^{-2} u X \frac{\partial^{3} x}{\partial x^{2} \partial z}
$$

which, from symmetry,

$$
\begin{aligned}
& =-\frac{a^{-2}}{2} \int_{t_{0}}^{t_{1}} d t \iint d S n X \frac{\partial}{\partial z}\left(\begin{array}{l}
\partial^{2} x \\
\partial_{x^{2}}^{2}
\end{array}+\frac{\partial^{2} X}{\partial y^{8}}\right) \\
& =-\frac{a^{-2}}{2} \int_{t_{0}}^{t_{1}} d t \iint d S n X\left(\frac{\partial \phi}{\partial z}-\frac{\partial \psi}{\partial z}\right) \\
& =-\frac{1}{2} \iint d \operatorname{Szn} X\left(-\frac{1}{a^{2} r^{3}}+\frac{1}{a^{3} c^{3} \rho^{3}}\right),
\end{aligned}
$$

where

$$
\gamma^{2}=x^{2}+y^{2}+z^{2}
$$

and

$$
\rho^{2}=c^{-2}\left(x^{9}+y^{2}\right)+a^{-2} z^{2} \text {. }
$$

The contribution from the other terms of $X_{1}, Y_{1}, Z_{1}, u_{1}, v_{1}, 2 v_{1}$ will, in like manner, be found to be

$$
\iint d S\left(-\frac{1}{a c^{2} \rho^{8}}(m w-u v)-\frac{X}{a^{2} y^{8}}(n z+m y)\right) .
$$

On expanding $v$ and $v v$ near the origin in the form

$$
v=v_{0}+x\left(\frac{\partial v}{\partial x}\right)_{0}+\ldots
$$

we find $\quad-\iint d S \frac{(m v-n v)}{a c^{2} \rho^{3}}=-\frac{4 \pi}{3}\left\{\left(\frac{\partial w}{\partial y}\right)_{0}-\left(\frac{\partial v}{\partial z}\right)_{0}\right\}$

$$
=-\frac{4 \pi}{3} a^{-2} X_{0} \text {. }
$$


We have also

and

$$
-\iint\left\{\frac{X}{a^{3} r^{3}}(n z+m y)-\frac{1}{2} \cdot \frac{X}{a^{2} r^{3}} n z\right\} d S=-2 \pi a^{-2} X_{0}
$$

$$
-\frac{1}{2} \iint d S \frac{z n X}{a^{3} c^{2} \rho^{3}}=-\frac{2 \pi a^{\cdot 2}}{3}-X_{0}
$$

Hence the integration over $S_{2}$ gives altogether $-4 \pi a^{-2} X_{0}$, when $t=0$.

We have next to consider the integration over the surface $S_{1}$. By making use of the following theorems we can integrate at once with respect to $t$. Let $U$ be any function of $t$ which does not become infinite for any real value of $t$.

$$
\int_{t_{0}}^{t_{1}} U \zeta\left(\frac{r}{a}+t\right) d t=\int_{-\eta_{0}}^{\eta_{1}}(U)_{t a-v / u} \zeta(t) d t=(U)_{t=-v / / a^{*}} .
$$

In a similar manner,

$$
\begin{aligned}
& \int_{t_{0}}^{t_{2}} U \frac{\partial \zeta\left(\frac{r}{a}+t\right)}{\partial r} d t=-\frac{1}{a}-\left(\frac{\partial}{\partial t}\right)_{t \ldots r / a}, \\
& \int U^{\partial^{2} \zeta\left(\frac{r}{a}+t\right)} \frac{1}{\partial r^{2}} d t=\frac{1}{a^{j}}\left(\frac{\partial^{2} U}{\partial t^{2}}\right)_{t \ldots+r / a}, \\
& \int \frac{\partial \zeta(\rho+t)}{\partial \rho} d t=-\left(\frac{\partial U}{\partial t}\right)_{t \cdot-\rho} .
\end{aligned}
$$

Thus of the terms in the surface integral, one set is formed at the time $t=-\frac{r}{a}$, whilst the other set is formed at the time $t=-\rho$. If the origin be outside the closed suiface $S_{1}$, it will be necessary to add a third boundary, an infinite sphere having its centre at the origin. Its contribution will vanish if we take the initial disturbance to be confined to the region $S_{1}$.

Taking a new origin so that the old origin becomes $x^{\prime}, y^{\prime}, z^{\prime}$, and reckoning $t$ from a different time, we can now write down the values of $X, Y, Z$ at the point $x^{\prime}, y^{\prime}, z^{\prime}$ and time $t$ in terms of a surface integral. The notation []$_{t-r, n},[]_{t-p}$ will be used to denote that the quantities inside the brackets are to be formed at the times $t-\frac{r}{a}$ and $t-\rho$ respectively; we shall also use the symbol

$$
\varpi^{9}=\left(x-x^{\prime}\right)^{3}+\left(y-y^{\prime}\right)^{8} .
$$




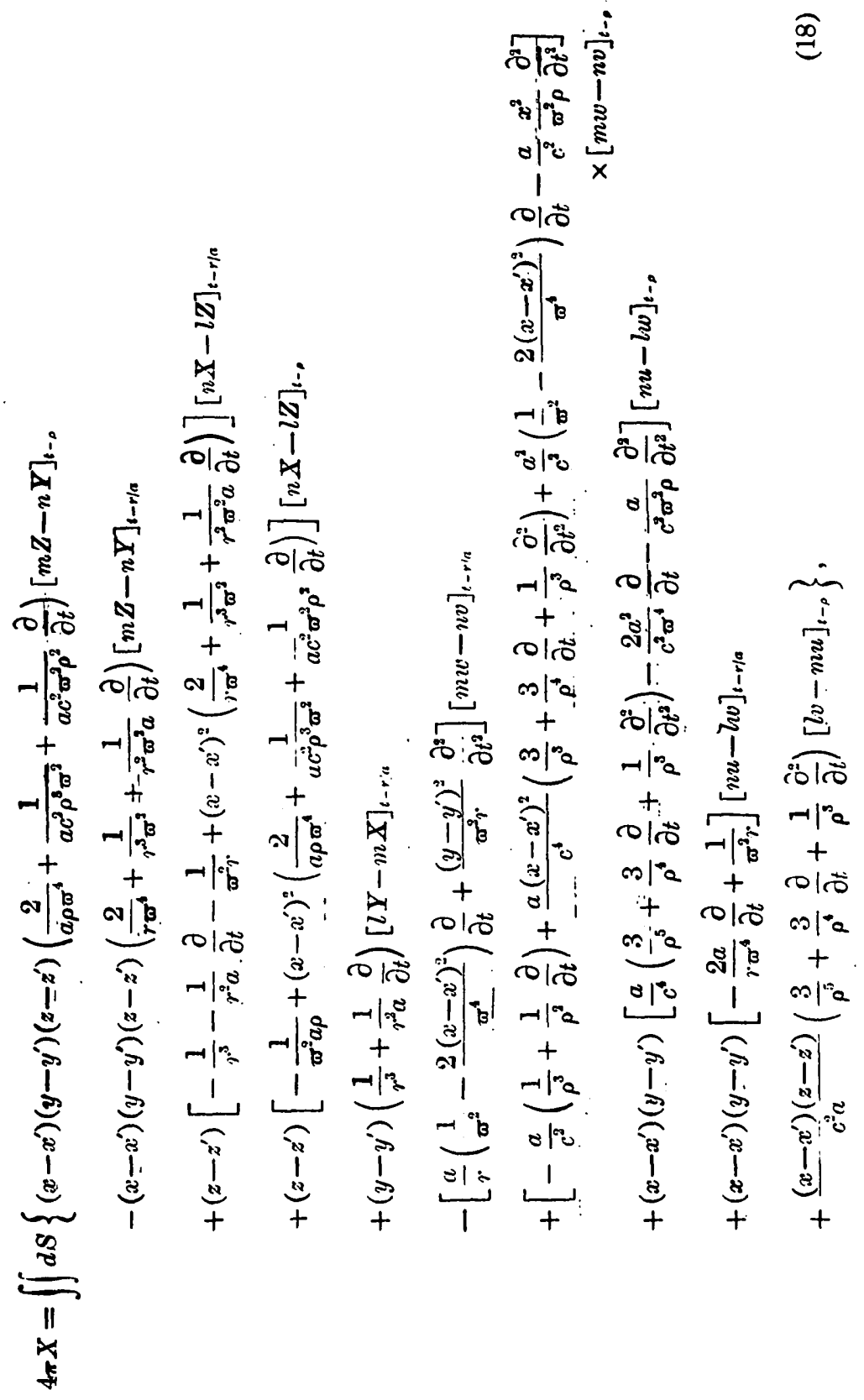




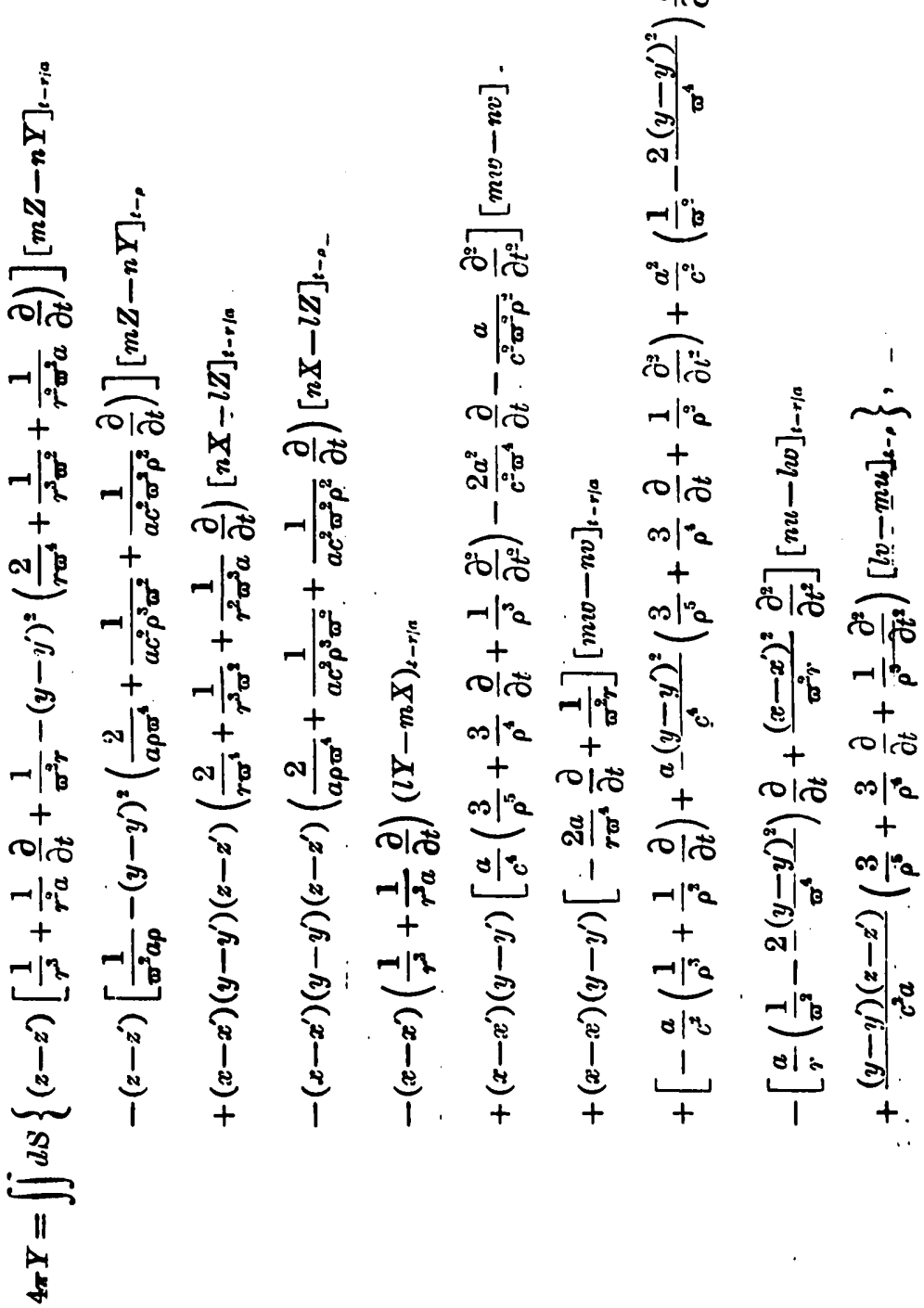

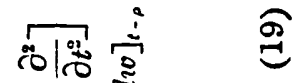

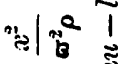

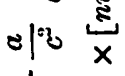$$
\text { वात्ठ }
$$

$-\left.1\right|^{\circ}$ 


$$
\begin{aligned}
& 4 \pi Z=\iint d S\left\{-\frac{\left(y-y^{\prime}\right)}{a c^{y}}\left(\frac{1}{\rho^{y}}+\frac{1}{\rho^{2}} \frac{\partial}{\partial t}\right)[m Z-n Y]_{t-\rho}\right. \\
& +\frac{\left(x-x^{\prime}\right)}{a c^{2}}\left(-\frac{1}{\rho^{3}}+\frac{1}{\rho^{2}} \cdot \frac{\partial}{\partial t}\right)[n X-l Z]_{l-\rho} \\
& +\underset{a c^{2}}{\left(x-a^{\prime}\right)\left(z-z^{\prime}\right)}\left(\underset{\rho^{5}}{3}+\frac{3}{\rho^{4}} \underset{\partial t}{\partial}+\frac{\partial}{\rho^{3}} \frac{\partial^{2}}{\partial t^{2}}\right)[m v-n v]_{i-\infty} \\
& +\underset{a c^{2}}{\left(y-y^{\prime}\right)\left(z-z^{\prime}\right)}\left(\begin{array}{c}
3 \\
\rho^{\prime}
\end{array}+\frac{3}{\rho^{4}} \frac{\partial}{\partial t}+\frac{1}{\rho^{3}} \frac{\partial q}{\partial t^{2}}\right)[n u-l w]_{t-\rho} \\
& +\left[+\frac{2}{a}\left(\frac{1}{\rho^{3}}+\frac{1}{\rho^{2}} \frac{\partial}{\partial \dot{t}}\right)\right. \\
& \left.\left.-\frac{\left(x-x^{\prime}\right)^{2}+\left(y-y^{\prime}\right)}{a c^{2}}\left(\frac{3}{\rho^{3}}+\frac{3}{\rho^{4}} \frac{\partial}{\partial t}+\frac{1}{\rho^{3}} \frac{\partial^{2}}{\partial t^{3}}\right)\right][l v-n i u]_{t-p}\right\} \text {. }
\end{aligned}
$$

Thus we have expressed the value of $X, Y, Z$ at a point $x^{\prime}, y^{\prime}, z^{\prime}$ in telms of a surface integral. This is the mualytical expression of the principle of Huygens. The disturbance at any point at a time $t$ is expressed in terms of the values of the electric force and magnetic displacement on any given surface at times $1-"$ " nnd $t-\rho$. In other words, each element of the surface crun he regarded us a source of clisturbance sending ont two waves, one with velocity $\iota$ and the other with velocity $\left\{\left(l^{3}+m^{2}\right) c^{-2}+n^{2} a^{-2}\right\}^{-1}$. Let us consider, for example, the parts of (18), (19), and (20) which inrolve (mZ $Z Y$ ) under the sign of integration. On examination they will be ound to differ only from the expression $X_{1}, Y_{1}, Z_{1},(7)$, by having $[m Z-n Y]_{t-p_{i n}}$ and $[m Z-n Y]_{i-p}$ in pluce of $\frac{\partial}{\partial t} H^{\prime}\left({ }^{\prime \prime}+t\right)$ and $\frac{\partial}{\partial t} F(\rho+t)$; so that these portions of the integral can be interpreted as the electric force due to a magnetic cloublet of moment proportional to $m Z-n Y$ and having its exis along the axis of $x$. Thaking the other terms in like manuer, we shall find that tre electric force at any point can be regarded as clue to a double system of sourees situater on the surfnce, one systen bcing electric doublets of moments proportional at ench point to the vector $m u-n v, m n-l n, 1 r-m n$, mal tho other sysstem being magnetic doublats of moments proportional to $m \%-n$ ), $n . \lambda-l Z, l Y-m X$. 


\section{The Direction of Vibration.}

If the components of electric force at any point due to a system of plane waves be denoted by $X_{0} f(l x+m y+n z-k t), Y_{0} f(l x+m y+n z-k t)$, $Z_{0} f(l x+m y+n z-k t)$ where $l^{2}+m^{2}+n^{2}=1$ and $X_{0}, Y_{0}, Z_{0}$ are independent of $x, y, z$, and $t$, then on substituting in equations (1) we find either

$$
\text { (I.) } k^{2}=c^{3}\left(l^{2}+n^{2}\right)+a^{2} n^{2}
$$

or

$$
\text { (1I.) } l^{2}=a^{2} \text {, }
$$

showing that the plane waves a.t any time $t_{-}$touch

$$
\text { (I.) } \frac{x^{2}}{c^{2}}+\frac{y^{2}}{c^{2}}+\frac{z^{2}}{a^{2}}=t^{2}
$$

or

$$
\text { (II.) } x^{9}+y^{2}+z^{2}=a^{2} t^{2} \text {. }
$$

'I'These are the extraordinary and ordinary wave surfaces respectively. It, will also be found that the direction of electric vibration (or electric displacement) is the trace of the radius vector on the tangent to the wave surface at the point where the radius vector meets it. 'This is the well known construction of Huygens and Fresnel for pline wave trains. In questions dealing with a divergent disturb:uce it is usually assumed that this construction holds also for such cases. But we shall now examine if any solution of the equations (1) exists infinite only at one point and having the electric displacement obeying this law of Huygens and Fresnel. We shall consider first an extraordinary wave diverging from a point. Putting.

$$
\omega^{2}=x^{2}+y^{2}
$$

we have to determine $U$ such that $\left\{\frac{x z}{\sigma^{3}} U, \frac{y z}{\sigma^{2}} U,-U\right\}$ are possible values for the components of electric force, and that they are infinite nnly at the origin. From these values, since

$$
a^{-2} \frac{\partial X}{\partial x}+a^{-2} \frac{\partial Y}{\partial y}+c^{-2} \frac{\partial Z}{\partial z}=0
$$

we have

$$
\frac{a^{-2}}{\varpi} \frac{\partial U}{\partial \sigma}=\frac{c^{-2}}{z} \frac{\partial U}{\partial z}
$$

therefore

$$
U=f\left(c^{-2} \sigma^{2}+a^{-2} z^{2}\right)=f(p), \text { say. }
$$




$$
\text { But } \begin{aligned}
\frac{\partial^{2} Z}{\partial x^{2}}+\frac{\partial^{2} Z}{\partial y^{2}}+\frac{\partial^{2} Z}{\partial z^{2}}-c^{-2} \frac{\partial^{2} Z}{\partial t^{2}} & =\frac{\partial}{\partial z}\left(\frac{\partial X}{\partial x}+\frac{\partial Y}{\partial y}+\frac{\partial Z}{\partial z}\right) \\
& =\left(1-\frac{a^{2}}{c^{2}}\right) \frac{\partial^{3} Z}{\partial z^{2}} ;
\end{aligned}
$$

therefore

$$
c^{2} \frac{\partial^{2} Z}{\partial x^{2}}+c^{2} \frac{\partial^{2} Z}{\partial y^{3}}+a^{2} \frac{\partial^{2} Z}{\partial z^{2}}=\frac{\partial^{2} Z}{\partial t^{2}}
$$

or

$$
\frac{1}{\rho} \frac{\hat{\sigma}^{2}}{\partial \rho^{2}}(\rho Z)=\frac{\partial^{x} Z}{\partial t^{2}}
$$

therefore

$$
\begin{aligned}
& Z=\frac{f_{1}(\rho-t)+f_{1}(\rho+t)}{\rho}, \\
& X=-\frac{d^{\prime} z}{\sigma^{2} \rho}\left\{f_{1}(\rho-t)+f_{2}(\rho+t)\right\}, \\
& Y=-\frac{y z}{\sigma^{2} \rho}\left\{f_{1}(\rho-t)+f_{3}(\rho+t)\right\} .
\end{aligned}
$$

Hence the only solutions we can find obeying Huygens' construction have $X$ and $Y$ infinite along the optic axis. These solutions, it may be seen, represent waves diverging from a source and running along a perfectly conducting wire extending along the axis of $z$ in both directions or else the disturbance due to a continuous stream of electrons being projected from the origin along the axis of $z$ with velocity $a . *$ We can find solutions for the ordinary wave, and they will also be infinite along the axis of $z$. It does not therefore seem legitimate to make any statement about the direction of vibration in a divergent beam close up to the source. It may be seen, however, from the forms (8), (9), and (10) given for simple singular solutions that at a rery great distance from the origin the vibration will satisfy the same laws as for plane waves. In $\$ 7$ an attempt is made to treat a problem in convergent light without any assumption as to the direction of vibration.

\section{The liay Direction.}

It is usually defined that the ray direction is the direction in which the energy of radiation, as defined by the Poynting flux, travels. We shall now consider if any solution exists which has a singularity only at the origin and in which the Poynting flux is radial in the 
immediate neighbourhood of the origin. Considering the case of frequency $p$, the most general solution is of the form

$$
\begin{aligned}
& X=\left(X_{0}+p X_{1}+p^{2} X_{2}+\ldots\right) e^{i p(t-v ; a)}+\left(X_{0}^{\prime}+p X_{1}^{\prime}+p^{2} X_{2}^{\prime}+\ldots\right) e^{i p(t-p)}, \\
& a=\left(a_{0}+p a_{1}+p^{2}\left(a_{2}+\ldots\right) e^{i p\left(t-v_{i}^{\prime} a\right)}+\left(a_{0}^{\prime}+p a_{1}^{\prime}+\ldots\right) e^{i p(t-\rho)},\right.
\end{aligned}
$$

with similar forms for $Y, Z, \beta, \gamma ; X_{0}, X_{0}^{\prime}, \ldots, a_{0}, a_{0}^{\prime}, \ldots$ are functions of $x, y$, and $z$. We can assume that the twelre tel'ms $X_{0}, X_{0}^{\prime}, Y_{0}, Y_{0}^{\prime}, Z_{0}, Z_{0}^{\prime}$, $a_{0}, a_{0}^{\prime}, \beta_{0}, i_{0}^{\prime}, \gamma_{0}, \gamma_{11}^{\prime}$ are not simultaneously zero. If they were, we could, by integrating with respect to $t$, restore the abore forms.

Since the Poynting flux is assumed to be radial,

$$
\begin{aligned}
x X+y Y+z Z & =0, \\
x u+y \beta+z \gamma & =0 .
\end{aligned}
$$

We have also

$$
\begin{aligned}
\iota^{-2} \frac{\partial X}{\partial x}+a^{-2} \frac{\partial Y}{\partial y}+c^{-2} \frac{\partial Z}{\partial z} & =0, \\
\frac{\partial \alpha}{\partial x}+\frac{\partial \beta}{\partial y}+\frac{\partial \gamma}{\partial z} & =0 .
\end{aligned}
$$

With respect to these relations the portions multiplying $e^{i p\left(e-r_{i n}\right)}$ and $e^{i p(t-\rho)}$ must separately vanish. For, since they must hold at any point for every value of $t$ on putting

$$
t=\frac{v}{11}+\frac{\pi}{2} \text { or }=\rho+\frac{\pi}{i},
$$

we can deduce the tbove result, and the results must also hold on putting $p=0$, i.e., making the period infinitely long.

Considering first $a_{11}, \beta_{11}, \gamma_{0}$, we have

$$
\begin{aligned}
\frac{\partial \gamma}{\partial y}-\frac{\partial \beta}{\partial z} & =\left(\frac{\partial \gamma_{0}}{\partial y}-\frac{\partial \beta_{0}}{\partial z}\right) e^{i p(1-v / n)}+\ldots=a^{-2} \dot{X} \\
& =n \text { quantity multiplied by } p ;
\end{aligned}
$$

therefor?

$$
\frac{\partial \gamma_{n}}{\partial y}=\frac{\partial \beta_{n}}{\partial z}
$$

Hence we can put $\quad a_{n}=\frac{\partial U}{\partial x}, \quad \beta_{0}=\frac{\partial U}{\partial y}, \quad i_{1}=\frac{\partial U}{\partial z}$

nnd.we find

$$
\begin{aligned}
& x \frac{\partial U}{\partial x}+y \frac{\partial U}{\partial y}+z \frac{\partial U}{\partial z}=[0, \\
& \frac{\partial^{2} U}{\partial x^{2}}+\frac{\partial^{2} U}{\partial y^{2}}+\frac{\partial^{2} U}{\partial z^{2}}=0 .
\end{aligned}
$$


$U$ therefore is independent of $r$, and depends only on the direction angles $\theta, \phi\left(\cos \theta=\frac{z}{r}, \sin \theta \sin \phi=\frac{y}{r}, \ldots\right)$. Putting $\tan \frac{1}{2} \theta \cos \phi=\xi$ $\tan \frac{1}{2} \theta \sin \phi=\eta$

we have

$$
\frac{\partial^{2} U}{\partial \xi^{2}}+\frac{\partial^{2} U}{\partial \eta^{2}}=0
$$

and $U$ is finite for all real values of $\xi$ and $\eta$. From this it follows that $U$ is a constant. Hence $\alpha_{i j}=0, \beta_{0}=0, \gamma_{0}=0, \ldots$; in like manner. $a_{0}^{\prime}=0, \beta_{0}^{\prime}=0, \ldots$, contrary to our hypothesis. Hence no solution exists in which the Poynting flux is radial and which has a singularity only as the origin. (The type of solution found in $\S 4$ can be shown to satisfy this condition.*).

\section{Application of Huygens' Principle to a Crystalline Medium vith n Plane Fice.}

Let us take the plane of $x y$ to be the surface of a crystalline medium, the positive direction of the $z$ axis coinciding with the axis of the crystal, and let us suppose the face of the crystal.to be disturbed by plane waves which travel in the outside medium with velocity $V$ and fall on the face in the direction $(0, \sin i, \cos i)$. If the values of electric force and magnetic displacement at the origin just inside the crystal be represented by the real parts of $\left(X_{0} e^{i j t}, Y_{0} e^{i j t}, Z_{0} e^{i, t t}\right)$ and $\left(u_{0} e^{i j t}, v_{0} e_{i, t}, v_{0} e^{i n t}\right)$ respectively, then the values $n t$ any other part of the face of the crystal will be $X_{0} e^{i / f\left[t-\left(\nu \sin i|| r^{*}\right)\right.}$,

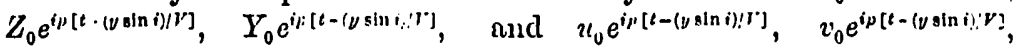
$u_{i n} e^{\left.i,(t-(y) \sin i) / r^{\prime}\right]}$.

With these values let us evaluate the integrals on the right-hand sides of (18), (19), and (20), the region of integration being an intinite rectangle on the plane ay with the origin as centre. We have

$$
\begin{aligned}
& l=0, \quad n=0, \quad n=1, \quad d S=d x d y, \\
& {[m Z-n Y]_{\ell-\rho}=-y_{0} e^{i p\left[t-\rho-(y \sin i) / r^{\cdot}\right]}, \ldots .}
\end{aligned}
$$

- It has been pointel out to mo by oue of tho referees that the results of $\$ \S 4$ and $\sigma$ amount to saying that the wave fronts (if defined us contrining in the tumgent, pline both the clertric rlimplasement and magnetio force) nenr a source nre not ellipsoidnl. 
If we write

$$
\begin{aligned}
& \phi^{\prime}=\frac{e^{-i p(v / a-t)}}{r}, \\
& \psi^{\prime}=\frac{a}{c^{\underline{a}}} \frac{e^{-i \mu(\rho-t)}}{\rho}, \\
& \chi^{\prime}=\int_{0}^{\varpi} \frac{a d \lambda}{i p \lambda}\left\{e^{-i p \sqrt{c^{-2} \lambda^{2}+a^{-2} z^{2}}+i p t}-e^{-i p / a \sqrt{\lambda^{2}+z^{2}}+i p t}\right\},
\end{aligned}
$$

it will be found that $\phi^{\prime}, \psi^{\prime}, \chi^{\prime}$ are connected by the same relations (2), (3), (4), and (5) as $\phi, \psi$, and $\chi$, and we can write the expressions for the electric force at any point $x^{\prime}, y^{\prime}, z^{\prime}$ in the crystal in the forms

$$
\begin{aligned}
& X=\frac{1}{4 \pi} \iint d x d y e^{-(i m, \sin i) \mid r}\left\{-\frac{\partial^{3} \chi^{\prime}}{\partial x \partial y \partial_{z}} Y_{0}+\left(\frac{\partial \varphi^{\prime}}{\partial z}-\frac{\partial^{3} \chi^{\prime}}{\partial x^{2} \partial z}\right) X_{0}\right. \\
& -\left(-\ddot{\phi}+c^{2} \frac{\partial^{3} \psi^{\prime}}{\partial x^{2}}+\frac{\partial^{2} \ddot{x}}{\partial x^{2}}\right) v_{0}+\left(c^{2} \frac{\partial^{2} \psi^{\prime}}{\partial x \partial y}+\frac{\partial^{2} \ddot{x}}{\partial x} \partial u_{y}^{\prime}\right), \\
& Y=\frac{1}{4 \pi} \iint \partial_{x} \partial y e^{-(i p y \sin i) / l}\left\{-\left(-\frac{\partial \phi^{\prime}}{\partial z}+\frac{\partial^{s} \chi^{\prime}}{\partial y^{2} \partial z}\right) Y_{0}-\frac{\partial^{8} \chi^{\prime}}{\partial x \partial y \partial z} X_{0}\right. \\
& \left.-\left(c^{2} \frac{\partial^{2} \psi^{\prime}}{\partial x \partial y}+\dot{\partial}^{2} \ddot{\chi^{\prime}} \partial \partial_{y}\right) v_{v}+\left(-\ddot{\psi}+c^{2} \frac{\partial^{2} \psi^{\prime}}{\partial y^{2}}+\frac{\partial^{2} \ddot{x}}{\partial y^{3}}\right) u_{0}\right\}, \\
& Z=\frac{1}{4 \pi} \iint d x d y e^{-(i m \sin i) \mid \cdot}\left\{-\frac{c^{9}}{a^{2}} \frac{\partial \psi^{\prime}}{\partial y} Y_{0}+\frac{c^{2}}{a^{2}} \frac{\partial \psi^{\prime}}{\partial x} X_{0}-c^{2} \frac{\partial^{2} \psi^{\prime}}{\partial x \partial z} v_{0}\right. \\
& \left.+c^{2} \partial^{\partial^{2} \psi} \partial_{z} u_{0}\right\}
\end{aligned}
$$

We shall now show that the integratious depend on the two integrals

We have

$$
\begin{aligned}
& \iint \phi e^{-(i p y \sin i) / V} d x d y=P(\text { say }), \\
& \iint \psi e^{-(\text {(pysin }) / V} d x d y=Q .
\end{aligned}
$$

$$
\begin{aligned}
& \iint \frac{\partial^{s} \chi^{\prime}}{\partial x \partial y \partial z} e^{-(i, y \sin i) / V} d x d y=\int\left[\frac{\partial^{z} \chi^{\prime}}{\partial y \partial z}\right]_{-x}^{\infty} e^{-(i p y \sin i) / V} d y=; 0 \\
& \iint \frac{\partial^{3} \chi^{\prime}}{\partial x^{2} \partial z} e^{-(i p y \sin i) / r} d x d y=0, \\
& \iint \frac{\partial^{2} \ddot{x}}{\partial x \partial y} e^{-(i p y \sin i) / v} d x d y=0,
\end{aligned}
$$




$$
\begin{aligned}
& \iint \frac{\partial \phi^{\prime}}{\partial z} e^{-(i p y \sin i) / r} d x d y=-\frac{\partial P}{\partial z^{\prime}}, \\
& \iint \ddot{\phi} e^{-(i p y \text { sin } i) / v} d x d y=\frac{\partial^{y} P}{\partial t^{2}} ; \\
& \iint\left(-\frac{\partial \phi^{\prime}}{\partial z}+\frac{\partial^{s} x^{\prime}}{\partial y^{8} \partial z}\right) e^{-(i j y \sin i) / r^{*}} d x d y \\
& =\iint\left(-\frac{\partial \psi^{\prime}}{\partial z}-\begin{array}{c}
\partial^{s} x^{\prime} \\
\partial x^{3} \partial z
\end{array}\right) e^{-(i p y \sin \phi) / r} d x d y=\frac{\partial Q}{\partial z^{\prime}}, \ldots ;
\end{aligned}
$$

so that the integrals become

$$
\begin{aligned}
& 4 \pi X=-X_{0} \frac{\partial P}{\partial z^{\prime}}+v_{0} \frac{\partial^{2} P}{\partial t^{2}}, \\
& 4 \pi Y=-Y_{0} \frac{\partial Q}{\partial z^{\prime}}+u_{0}\left(-\frac{\partial^{2} Q}{\partial t^{2}}+c^{2} \frac{\partial^{2} Q}{\partial y^{\prime 2}}\right), \\
& 4 \pi Z=+Y_{0} \frac{c^{2}}{a^{2}} \frac{\partial Q}{\partial y^{\prime}}+u_{0} c^{2} \frac{\partial^{2} Q}{\partial y^{\prime} \partial z^{\prime}} \\
& P=\int_{-\infty}^{\infty} \int_{-\infty}^{\infty} \frac{e^{-i \mu\left(\frac{\dot{y}}{1}+\frac{y \sin i}{r}-t\right)}}{r} d x d y \text {. }
\end{aligned}
$$

Transform the variable $a$, thus

$$
x-x^{\prime}=\frac{a}{2}\left(\xi-\frac{\varpi^{2}}{a^{2} \xi}\right)
$$

so that

$$
q^{3}=\left(x-x^{\prime}\right)^{2}+\sigma^{2}=\frac{a^{2}}{4}\left(\xi+\frac{\sigma^{2}}{a^{2} \xi}\right)^{2},
$$

where

$$
\varpi^{2}=\left(y-y^{\prime}\right)^{2}+z^{\prime 2} \text {. }
$$

Then

$$
P=\int_{-\infty}^{\infty} d y \int_{0}^{\infty} \frac{d \xi}{\xi} e^{-i p\left(\frac{\xi}{2}+\frac{\Phi^{2}}{2 a^{2} \xi}+\frac{y \sin i}{\gamma}-t\right)} .
$$

Making use of the theorem

$$
\int_{-\infty}^{\infty} e^{-i a)^{2}} d y=e^{-i i \pi} \sqrt{\frac{\pi}{a}}
$$

(where $\alpha$ is real and positive), and putting $\xi=\eta^{2}$, we get

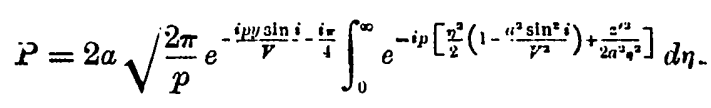

But

$$
\int_{0}^{\infty} e^{-i\left(a x^{2}+\beta / x^{3}\right)} d x=\frac{1}{2} \sqrt{\frac{\pi}{a}} e^{-2 i \alpha \beta-}
$$


if $\boldsymbol{a}$ and $\beta$ are real and positive. We get, findlly,

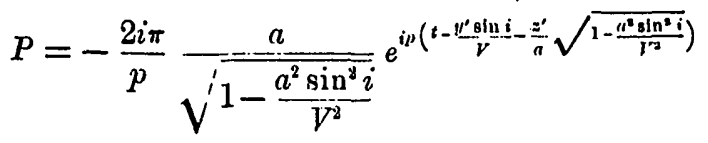

We may notice that, if we integrate first with respect to $x$, we get

$$
P=\int_{-\infty}^{\infty} K_{0}\left(\frac{i p \omega}{a}\right) e^{-i \mu\left(\frac{\nu \sin i}{r}-t\right)} d y
$$

in the usual notation of Bessel functions. In fact we are replacing the point-sources by line-sources. In like manner, we find

$$
Q=-\frac{2 i \pi}{p} \frac{a}{\sqrt{1-\frac{a^{2} \sin ^{2} i}{V^{2}}}} e^{i p\left(t-\frac{\nu^{\prime} \sin i}{V}-\frac{z^{\prime}}{a} \sqrt{\left.1-\frac{c^{2} \sin ^{1} i}{r^{2}}\right)} .\right.}
$$

Using these values, we get

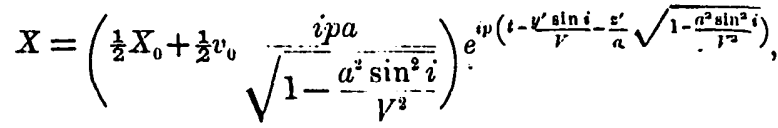

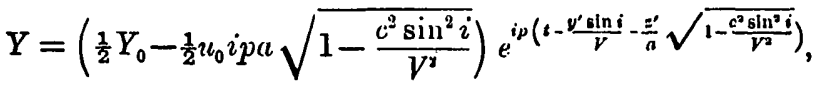

$$
\begin{aligned}
& Z=\frac{c^{2}}{a^{2}}\left(-\frac{1}{2} Y_{0} \frac{a \sin i}{V_{i} \sqrt{1-\frac{c^{3} \sin ^{2} i}{V^{2}}}}+\frac{1}{2} u_{0} \frac{i p a^{2} \sin i}{V}\right) e^{i p\left(t-\frac{L^{2} \sin i-z^{2}}{r} \sqrt{1-\frac{c^{2} \sin ^{2}}{r^{2}}}\right.}
\end{aligned}
$$

The quantities $\mathrm{X}_{0}, Y_{0}, u_{0}, v_{0}$, caused by two trains of waves, one incident and the other reflected, are not independent. We can in this case find the relation between then by noticing that the above expressions for $X$ and $Y$ must be equal to $X_{0}$ and $Y_{0}$ at the origin. This leads to the following simplified expressions :-

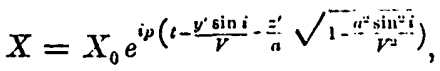

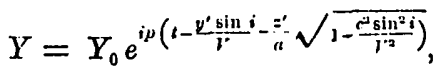

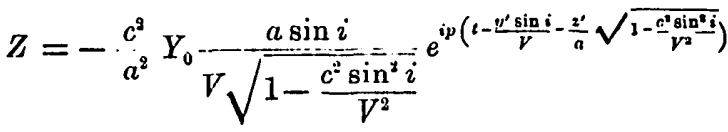

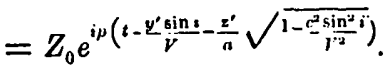


The disturbance in the crystal thus consists of two parts, one refracted at an angle $r$ given by

$$
\sin r=\frac{a}{V} \sin i
$$

the vibration (i.e., electric displacement) being perpendicular to the plane of incidence and travelling with a velocity $a$, the other refracted at an angle $r^{\prime}$ given by

$$
\sin r^{\prime}=\frac{a}{V} \sin i\left\{1+\frac{a^{2}-c^{3}}{V^{3}} \sin ^{2} i\right\}^{-1}
$$

with velocity $a\left\{1+\frac{a^{2}-c^{2}}{V^{2}} \sin ^{2} i\right\}^{-1}$, the vibration being in the plane of incidence. It is to be noticed that the electric donblets and the magnetic doublets which we imagine to be on the surface each contribute half the total amount of the disturbance.

\section{The Passage of Plane Waves through a Thin Crystalline Plate.}

If we consider any set of plane waves which fall on a plate of crystal with parallel sicies; the axis of the crystal being normal to both faces, on reaching the crystal a reflected system is set up, whilst the remainder is divided into two plane-polarized beams, each of which gives rise to reflected benms on reaching the otler face of the crystal, whilst we get finally an emergent beam travelling in the same direction as the incident beam. It is unnecessary to consider the effect of multiple reflections if the plate we are considering is taken to be much thicker than those which give rise to the phenomena of interference analogous to Newton's lings. The emergent beam consists of two parts in difterent phases. In fact, we nay easily see from the equations above that the difference in phase is

$$
\delta=\frac{p d}{a}\left\{\sqrt{1-\frac{c^{2} \sin ^{2} i}{V}}-\sqrt{1-\frac{a^{2} \sin ^{2} i}{V}}\right\},
$$

where $d$ is the thickness of the crystal; the amplitudes of the electric displacement in those two beams may be shown to be nearly proportional to the parts of the incident vibration resolved in and perpendicular to the plane of incidence (Preston's Light, chap. xiii.). Making use of these considerations, we find that a train of plane waves travelling in the direction $l, m, n$ and having the trace of the voL. Xxxv.-N0. 805 . 
vibration on the plane $x y$ parallel to the axis of $x$ will, after traversing the crystalline plate, yield components of electric force given by

$$
\begin{aligned}
& X=-n \frac{l^{2}+m^{8} e^{i \delta}}{l^{2}+m^{2}} G e^{i p[t-(l x+m y+n z) / V]}, \\
& Y=-n \frac{\ln \left(1-e^{i s}\right)}{l^{8}+m^{2}} G e^{i p[t-(l x+m y+m z) / m]}, \\
& Z=l G e^{i p[t-(l x+m y+m z) / V]}
\end{aligned}
$$

where $G$ is independent of $x, y, z$, and $t$, and

$$
\begin{gathered}
\delta=\frac{p d}{a}\left\{\sqrt{1-\frac{c^{2} \sin ^{2} i}{V}}-\sqrt{1-\frac{a^{2} \sin ^{2} i}{\ddot{V}}}\right\}, \\
\sin i=\sqrt{1-n^{2}} .
\end{gathered}
$$

\section{The Passage of a Divergent Bearn of Plane-polarized Light through a T'hin Crystalline Plate.}

It is an easy deduction from the fundamental electro-magnetic equations in free æther that no solution exists infinite at a point and having the electric force in a given direction. E.T. Whittaker (Brit. Assoc. Rep., 1902) has shown that any solution of these equations can be made up of a series of plane waves. We shall consider a plane-polarized divergent beam to consist of an infinite series of plane waves having their vibrations when resolved along the face of the crystal parallel to a fixed direction, which we may take to be the axis of $a$. Taking the origin on the side from which the light is coming, and the axis of $z$ parallel to the optic axis which is parallel to both faces, we get for the emergent beam

$$
X=\iint-n \frac{l^{2}+m^{2} e^{i \delta}}{l^{2}+m^{3}} G e^{i p[t-(l x+m y+n z) / n]} d \omega,
$$

with similar values of $Y$ and $Z$ where, to secure generality, we take $G=f(l, m, n)$ to be an arbitiary function of $l, m, n$, and $d \omega$ is the element of solid angle. The region of integration is determined by the aperture of the incident beam, and does not depend on $x, y$, or $z$. Here

$$
-n \frac{l^{4}+m^{2} e^{i \delta}}{l^{2}+m^{2}}=-n\left(l^{4}+m^{2} e^{i \delta}\right)\left(1+n^{2}+n^{4}+\ldots\right),
$$

whilst $\delta$ can be expanded in positive integral powers of $n$. If we replace $l, m, n$ by $-\frac{V}{i p} \frac{\partial}{\partial x},-\frac{V}{i p} \frac{\partial}{\partial y},-\frac{V}{i p} \frac{\partial}{\partial z}$, and suppose $\delta^{\prime}$ to be 
what $\delta$ becomes when we put $-\frac{V}{i p} \frac{\partial}{\partial z}$ for $n$, we get

$$
\begin{aligned}
X=f\left(-\frac{V}{i p} \frac{\partial}{\partial x},-\frac{V}{i p} \frac{\partial}{\partial y},-\frac{V}{i p} \frac{\partial}{\partial z}\right) \frac{V}{i p} \frac{\partial}{\partial z}\left(\frac{V^{2}}{p^{2}} \frac{\partial^{g}}{\partial x^{2}}+\frac{V^{2}}{p^{2}} \frac{\partial^{8}}{\partial y^{2}} e^{i s}\right) \\
\\
\times\left(1-\frac{V^{2}}{p^{2}} \frac{\partial^{2}}{\partial z^{2}}+\frac{V^{4}}{p^{4}} \frac{\partial^{4}}{\partial z^{4}}-\ldots\right) I
\end{aligned}
$$

where

$$
I=\iint e^{i p[t-(l x+m y+n z) / V]} d \omega .
$$

To find $I$, let $\quad l x+m y+n z=r \cos \theta$,

and let $\phi$ be the angle made by any plane through the direction $\frac{x}{r}, \frac{y}{r}, \frac{z}{r}$ a fixed plane, so that

$$
d \omega=\sin \theta d \theta d \phi .
$$

Then $\quad I=\iint e^{i p[t-(r \cos \theta) / V]} \sin \theta d \theta d \phi$

$$
=-\frac{2 \pi V}{i p} \frac{e^{i p(t-r / V)}}{r}+a \text { term independent of } x, y \text {, and } z \text {. }
$$

If we now consider points at a considerable distance from the origin, we have

$$
\begin{aligned}
-\frac{V}{i p} \frac{\partial I}{\partial x} & =\frac{x}{r} I, \\
-\frac{V}{i p} \frac{\partial I}{\partial y} & =\frac{y}{r} I, \\
-\frac{V}{i p} \frac{\partial I}{\partial z} & =\frac{z}{r} I, \\
-V^{2} \frac{\partial^{2}}{\partial x^{2}} I & =\frac{x^{2}}{r^{3}} I, \ldots .
\end{aligned}
$$

Now, putting $\lambda, \mu, \nu$ for $\frac{x}{r}, \frac{y}{\gamma}, \frac{z}{r}$, we get finally (on writing $\nu$ for $n$ in the values of $\delta$ )

$$
\begin{aligned}
& X=-v \frac{\lambda^{2}+\mu^{2} e^{i s}}{\lambda^{2}+\mu^{2}} \cdot f(\lambda, \mu, \nu) I, \\
& Y=-v \frac{\lambda \mu\left(1-e^{i s}\right)}{\lambda^{2}+\mu^{2}} f(\lambda, \mu, \nu) I, \\
& Z=\lambda f(\lambda, \mu, \nu) I .
\end{aligned}
$$


244. On the Propagation of Light in a Uniaxal Orystal. [Nov. 13,

These are the expressions for the electric force due to a beam of divergent plane-polarized light which has passed through a thin plate. For directions near the axis of $Z$ we can neglect $Z$, and, if we suppose an analyzer to be an instrument which allows to pass only vibrations in a certain plane, we can find an expression for the resultant analyzed vibiation which will coincide with the usual expression given in text-books of physical optics. For instance, if the principal planes of the analyzer and polarizer are at right angles, the resulting vibration is given by

$$
Y=-v \frac{\lambda \mu\left(1-e^{i \delta}\right)}{\lambda^{8}+\mu^{8}} f(\lambda, \mu, v) I
$$

and $f(\lambda, \mu, v)$ never vanishes, as the plate is taken of a certain thickness. We have then a black cross given by $\lambda=0, \mu=0$, and a series of rings given by $\left(1-e^{i s}\right) I=0$.

\section{Note on Huygens' Principle.}

Prof. Love, in his paper already quoted, has discussed the question of the intensity at any point of the wave front of a secondary wavelet as used in Huygens' principle. The question is indeterminate, and in this note I propose to point out the relationship between the various solutions.

In the case of an isotropic medium, putting $a=c=l^{r}$ and taking the incident radiation to be given by

$$
\begin{gathered}
X=X_{0} e^{i p, i=\xi l v)}, \quad Y=0, \quad Z=0 \\
u=0, \quad v=-\frac{i X_{0}}{p V} e^{i p(t-z / b)}, \quad v=\frac{i X_{0}}{p V} e^{i p(t-s / V)}
\end{gathered}
$$

Putting

$$
\Phi=\frac{e^{i p r i r}}{r}
$$

then the values of $X, Y, Z$ at a point $x^{\prime}, y^{\prime}, z^{\prime}$ on the positive side of the plane of $x y$ are given by

$$
\begin{aligned}
& 4 \pi X=\iint d x d y X_{0} e^{i p(t-s / V)}\left\{\frac{\partial \Phi}{\partial z}+\frac{i}{p V}\left(-\ddot{\Phi}+V^{z} \frac{\partial^{2} \Phi}{\partial x^{2}}\right)\right\}, \\
& 4 \pi Y=\iint d x d y X_{0} e^{i p(t-s / V)} \frac{i V}{p} \frac{\partial^{2} \Phi}{\partial x \partial y} \\
& 4 \pi Z=\iint d \ddot{x} d y X_{0} e^{i p(t-s / V)}\left[\frac{\partial \Phi}{\partial x}+\frac{i V}{p} \frac{\partial^{2} \Phi}{\partial x \partial z}\right] .
\end{aligned}
$$


The terms $\frac{\partial \Phi}{\partial z}$ and $\frac{\partial \Phi}{\partial x}$ are due to magnetic doublets, whilst the others are due to electric doublets. All these integrals vanish if $x^{\prime}, y^{\prime}, z^{\prime}$ be on the negative side of $(x, y)$ and $\frac{\partial \Phi}{\partial z}$ and $\frac{\partial^{2} \Phi}{\partial x \partial z}$ change sign on crossing the plane a $y$; hence the electric and magnetic doublets each contribute half the total amount of the magnetic force. Putting

$$
\lambda=\frac{x^{\prime}-x}{r}, \quad \mu=\frac{y^{\prime}-\eta}{r}, \quad \nu=\frac{z^{\prime}}{r},
$$

we find that at a great distance the electric force contributed by a magnetic doublet is proportional to $(-\nu, 0, \lambda)$, whilst the electric force contributed by an electric doublet is proportional to $\left[-\left(\mu^{8}+v^{2}\right), \lambda \mu, \lambda \nu\right]$. If we take the sources as they stand in tine integrals above, we find that the electric force contributed by an element of the surface varies as $1+v$. 'I'his is Prof. Love's expression. If we took only' the magnetic doublets, the force would vary as $\sqrt{v^{2}+\lambda^{2}}$. If we took only the electric doublets, we should get Lord Rayleigh's form $\sqrt{\mu^{3}+\nu^{2}}$. Since $X, Y, Z$ are independent of $x, y, z$, then

$$
\frac{\partial X}{\partial z}=0, \quad \frac{\partial Y}{\partial z}=0, \quad \frac{\partial Z}{\partial z}=0 .
$$

This means that a certain system of electric quadruplets distributed on the surface have a null effect. Combining these sources with the electric doublets, we find Sir G. Stokes's expression $\sqrt{\mu^{2}+\nu^{2}}(1+\nu)$. The region of integration is supposed to be an infinite rectangle.

Sets of Intervals on the Straight Line. By W. H. Yousa. Received October 4th, 1902. Read November 13th, 1902.

The consideration of the theory of linear sets of points leads, in a natural manner, to that of sets of intervals on a straight line. Indeed, in some respects it is more natural to begin with the latter than with the former. For example, every set of non-overlapping 